\title{
Non-covalent immobilization of chiral copper complexes on Al- MCM41: effect of the nature of the ligand
}

\author{
Robert A. Feldman, José M. Fraile*
}

Instituto de Síntesis Química y Catálisis Homogénea (ISQCH), Facultad de Ciencias, C.S.I.C. - Universidad de Zaragoza, E-50009 Zaragoza, Spain. E-mail:

jmfraile@unizar.es

\begin{abstract}
$\mathrm{Cu}-\mathrm{Al}-\mathrm{MCM} 41$, prepared by deposition of copper(II) triflate with incipient wetness impregnation and thermal treatment under air flow, can be modified with different chiral ligands of the bis(oxazoline) family. The efficiency of the supported chiral catalysts in the enantioselective cyclopropanation of styrenes with ethyl diazoacetate depends on the nature of the ligand. The azabis(oxazolines) perform much better than the bis(oxazolines) and give stable catalysts that can be used for at least five consecutive runs, with productivities that can reach values close to 1000 molecules of cyclopropane per copper site. The best enantioselectivities obtained with these catalysts are in the range of $60-70 \%$ ee in the reaction at $90^{\circ} \mathrm{C}$.
\end{abstract}

Keywords: asymmetric catalysis; immobilized catalysts; mesoporous materials; bis(oxazolines); copper; cyclopropanation 


\section{Introduction}

The immobilization of chiral catalysts on solid supports is a way to combine the activity and selectivity advantages of homogeneous chiral catalysts with benefits associated with having an additional phase [1], however, the practical advantages in the catalytic process need to be sufficiently high in order to compensate the costs associated to the immobilization process [2]. A consideration with the covalent immobilization of chiral ligands is that it can require additional functionalization but this is not the case with non-covalent immobilization methods [3], when the essence if to have simple preparation procedures that utilize the same ligand used in homogeneous catalysis. The electrostatic interaction between the support and the catalytic complex is an ideal immobilization method, as it is held together with the strongest type of interactions, and its potential has been demonstrated in a good number of examples using different supports, such as organic polymers [3,4] or clays [3,5].

Silica based mesoporous materials with isomorphous substitutions in the framework are described to interact electrostatically with cationic chiral complexes $[3,6]$.

Chiral bis(oxazoline) ligands have been widely used in homogeneous catalysis [7], in many cases in the form of cationic complexes, and this permits them to be immobilized through electrostatic interactions. A rich body of literature describes work with supports such as clays [8-10] and nafion-silica composites [11], and a few examples with ordered mesoporous materials [12-16]. In our previous work, we compared different approaches to the electrostatic immobilization of bis(oxazoline)-copper complexes on Al-MCM41, and found that a direct cationic exchange is not easy to accomplish, and that an incipient wetness impregnation method leads to better results in the cyclopropanation reaction of styrene with ethyl diazoacetate [17]. A higher enantioselectivity and recoverability was obtained with a different approach to immobilizing the copper centres on the support, that utilized a three-step immobilization method, consisting of a first impregnation with copper(II) triflate, subsequent thermal treatment of the solid and final modification with the chiral ligand [18]. In this paper we demonstrate how this methodology performs with different bis(oxazoline) and azabis(oxazoline) [19] ligands (Figure 1). 
<smiles>[R]O[R6]</smiles>

$\left(\mathrm{R}=\mathrm{Ph},{ }^{\mathrm{i}} \mathrm{Pr},{ }^{\mathrm{t}} \mathrm{Bu}\right)$<smiles>[R]C1COC(N(C)C2=NC([R])CO2)=N1</smiles>

azaR

Figure 1. Ligands used in this work.

\section{Experimental}

\subsection{Preparation of the catalysts}

Al-MCM41 $(\mathrm{Si} / \mathrm{Al}=10)$ was prepared from sodium aluminate and fumed silica in a basic medium with cetyl trimethyl ammonium as the template and 1,3,5trimethylbenzene added to expand the pore size [20]. As previously described [18], AlMCM41 (500 mg) was added to a solution of copper(II) triflate (42 mg, $0.1 \mathrm{mmol}$ ) in $600 \mu \mathrm{L}$ of methanol and the container was shaken to ensure that the solution was evenly distributed. This material was then calcined $\left(1^{\circ} \mathrm{C} / \mathrm{min}\right.$ heating until $450^{\circ} \mathrm{C}$, maintained for $4 \mathrm{~h}$ ) under a $100 \mathrm{~mL} / \mathrm{min}$ flow of air to obtain what we designate "Cu-Al-MCM41". $\mathrm{Cu}-\mathrm{Al}-\mathrm{MCM} 41(500 \mathrm{mg}, 0.1 \mathrm{mmol} \mathrm{Cu})$ was added to a solution of the corresponding chiral ligand $(0.2 \mathrm{mmol})$ in $600 \mu \mathrm{L}$ of dichloromethane, the container was shaken to ensure that the ligand was evenly distributed, and this supported complex was then immediately used as a catalyst.

\subsection{Catalytic tests}

\subsubsection{Homogeneous cyclopropanation.}

At room temperature, EDA (2.5 mmol, $285 \mathrm{mg})$ dissolved in dichloromethane $(0.5 \mathrm{~mL})$ was added over the course of $2 \mathrm{~h}$ with a syringe pump to a solution containing the copper catalyst $(0.025 \mathrm{mmol}), \mathrm{n}$-decane $(100 \mathrm{mg})$ and alkene (styrene or $\alpha$-methyl styrene, $2 \mathrm{~mL}$ ). After the addition, the reaction was left to stir for $30 \mathrm{~min}$ and then analysed for the reaction yield and selectivity by CG. 


\subsubsection{Heterogeneous cyclopropanation.}

The supported catalyst $(100 \mathrm{mg}, 0.02 \mathrm{mmol} \mathrm{Cu}$ ) was suspended in the alkene (styrene or $\alpha$-methyl styrene, $2 \mathrm{~mL}$ ) with $\mathrm{n}$-decane $(100 \mathrm{mg})$ and the mixture was heated at $90^{\circ} \mathrm{C}$. EDA $(2.5 \mathrm{mmol}, 285 \mathrm{mg})$ dissolved in dichloromethane $(0.5 \mathrm{~mL})$ was added over the course of 15 min with a syringe pump. After the addition, the catalyst was separated by centrifugation, the products were analyzed by $\mathrm{GC}$, and the next reaction was performed by immediately re-suspending the catalyst in the alkene.

\section{Results and discussion}

The $\mathrm{Cu}-\mathrm{Al}-\mathrm{MCM} 41$ catalyst was prepared following the conditions described in our most recent paper [18]. The chosen support was an Al-MCM41 with a Si/Al molar ratio of 10, and with expanded porosity obtained by introduction of 1,3,5-trimethylbenzene as co-template, to yield a solid characterized to have an internal volume of $1.2 \mathrm{~mL} / \mathrm{g}$, BET surface area of $628 \mathrm{~m}^{2} / \mathrm{g}$, and BJH pore diameter of $92 \AA$. Copper was introduced by incipient wetness impregnation of $\mathrm{Cu}(\mathrm{OTf})_{2}$, and the solid was calcined at $450^{\circ} \mathrm{C}$ under an air flow with the view to produce a thermodynamically more stable interaction with the support and keeping the oxidation state +2 in well isolated copper centres, as shown by SEM-EDX. The copper may then be modified by incipient wetness impregnation with different chiral bis(oxazoline) and azabis(oxazoline) ligands (Figure 1), noting that the nature of both the central bridge (isopropylidene or methylimino) and the substituent in the oxazoline ring (phenyl, isopropyl, tert-butyl) have an influence on the stability of the resulting copper complex [21].

The supported chiral catalysts were first tested in the cyclopropanation of styrene (Scheme $1, \mathrm{R}=\mathrm{H}$ ) with ethyl diazoacetate, using an excess of styrene as solvent at $90^{\circ} \mathrm{C}$, which are the best conditions found in our previous work [18]. At $90^{\circ} \mathrm{C}$ in the homogeneous phase, cationic polymerization of styrene rapidly occurs and so the homogeneous reactions were carried out at room temperature. This difference in temperature needs to be taken into consideration when comparing the results, which are summarized in Figure 2. 


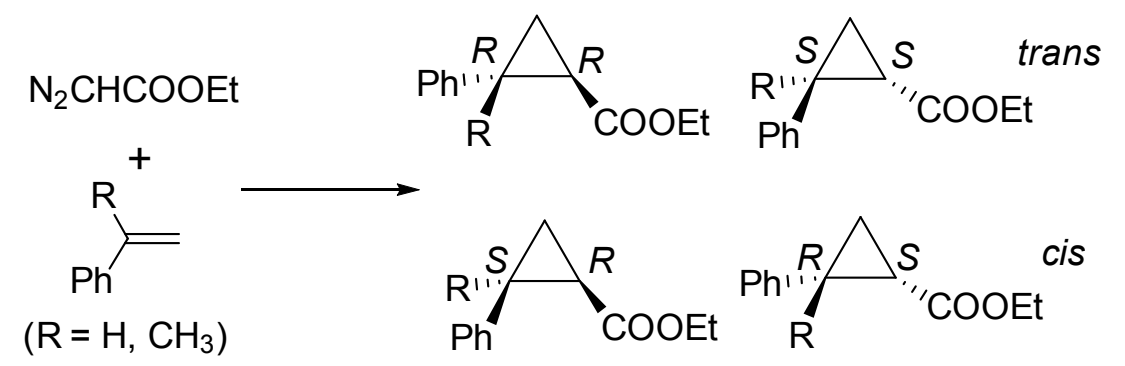

Scheme 1. Cyclopropanation of styrene and $\alpha$-methylstyrene with ethyl diazoacetate.
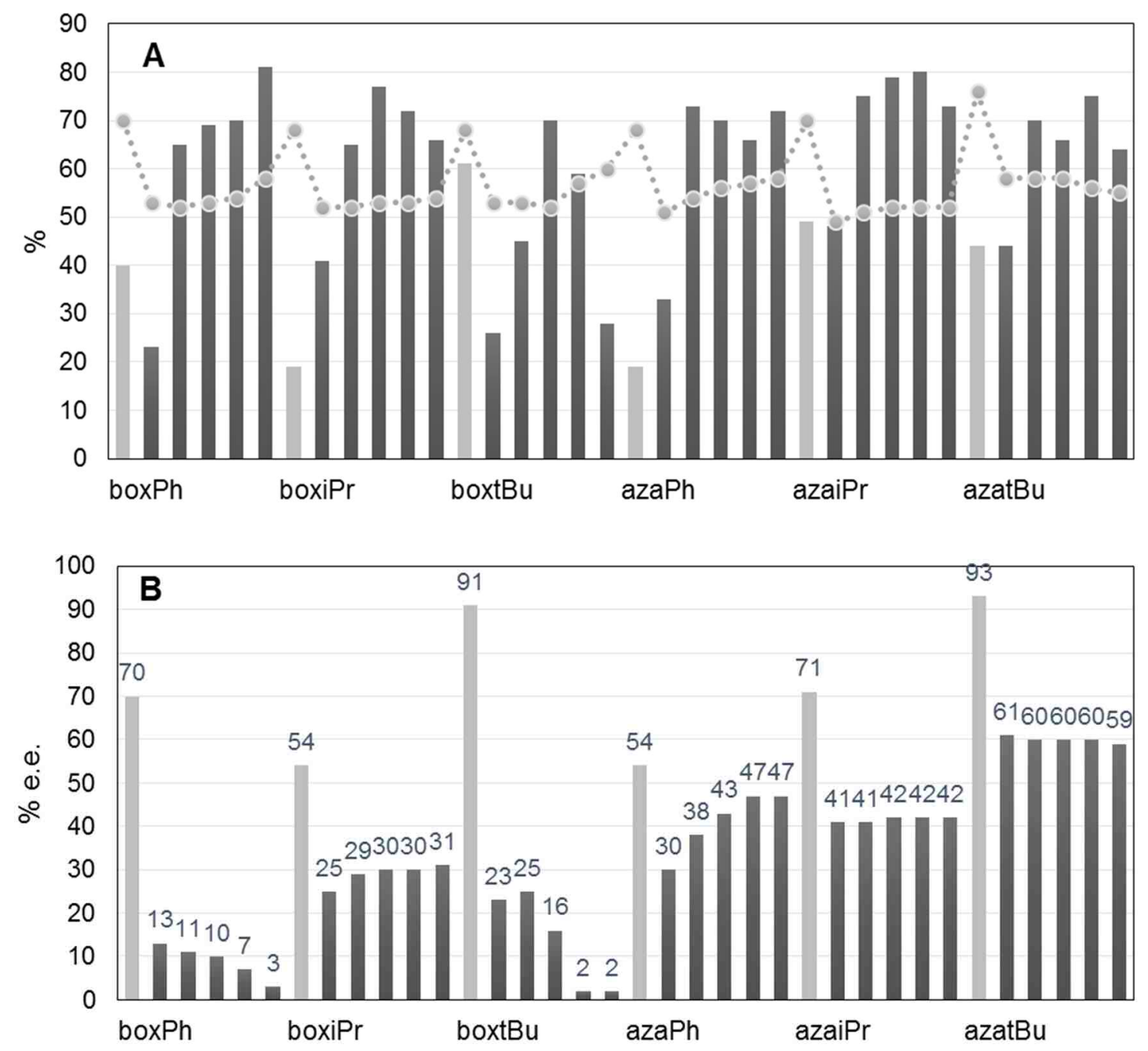

Figure 2. Results of the cyclopropanation of styrene with ethyl diazoacetate with the six different chiral ligands: A) yield of cyclopropanes in homogeneous phase (grey bars) and in heterogeneous phase (black bars) in succesive runs, and \% of trans cyclopropanes (grey dots), B) enantioselectivity (\% e.e.) of the trans cyclopropanes. 
In all cases the yield of cyclopropanes (Figure 2A, bars) was better than in the homogeneous phase, at least from the second run. An overall trend is that the yield is consistently lower in the first run than in the second run, and this may be attributed to a build up of to $\mathrm{Cu}(\mathrm{I})$ sites due to the reduction of $\mathrm{Cu}(\mathrm{II})$ to $\mathrm{Cu}(\mathrm{I})$ sites with diazoacetate. Remarkably, the reduced $\mathrm{Cu}(\mathrm{I})$ sites seem to be stable upon recycling, which can be inferred from the similar yields that were in the range of $65-80 \%$, in runs $2-5$. The only exception to this behaviour is the catalyst modified with box ${ }^{t} \mathrm{Bu}$, which reaches a maximum yield in the third run and is then deactivated in the successive reactions. The diastereoselectivity to the trans cyclopropane is also consistently lower in the case of the heterogeneous catalysts (Figure 2A, dots). The trans/cis ratio in solution varies from 68:32 to $76: 24$, but the modified Cu-Al-MCM41 catalysts lead to mixtures with ratios close to 50:50. Interpreting this behaviour would require one to be able to delineate whether this is due to a confinement effect imparted by the support or it is due to the higher reaction temperature.

Enantioselectivities (Figure 2B) are consistently lower for the heterogeneous catalysts, compared to the analogous homogeneous phase catalysts, and again, one could attribute this to either a confinement or temperature effect. Moreover, there are two separate trends of behaviour depending on the chiral ligand used. With the three azabis(oxazolines) and box ${ }^{i} \operatorname{Pr}$ the enantioselectivity values are moderate and stable upon recycling, with values around $60 \%$ e.e. occuring for $\mathrm{aza}^{\mathrm{t}} \mathrm{Bu}$, as the best result. Even in the case of azaPh the enantiomeric excess increases until it reaches a stable value of $47 \%$ e.e. in the fourth run, and this begs the question as to whether some kind of re-distribution of the ligand occurs during the initial reactions. The ${ }^{i}$ Pr-substituted ligands lead to enantioselectivities around 40 and 30\% e.e. for aza and bis(oxazoline), respectively. On the contrary, with box $\mathrm{Ph}$ and box ${ }^{\mathrm{t}} \mathrm{Bu}$ the enantioselectivities are already low in the first run and decreases upon reuse. It seems plausible that these differences in behaviour can be attributed to the different stabilities of the ligand- $\mathrm{Cu}$ complexes, which are higher for azabox as demonstrated by theoretical calculations [21] and the better performance and recoverability when immobilized by electrostatic interactions on clays and nafion-like solids [22], in ionic liquid phases [23] or by covalent grafting to organic polymers [24]. Even this higher stability has been pointed out as the reason for the better performance of azabis(oxazoline)-metal complexes as catalysts in other reactions, such as the enantioselective reduction of unsaturated esters 
with cobalt catalysts, both in homogeneous phase [25] and in recyclable systems [26]. Following this same reasoning, it appears that the box ${ }^{i} \mathrm{Pr}-\mathrm{Cu}$ complex is more stable than the boxPh-Cu and box ${ }^{t} \mathrm{Bu}-\mathrm{Cu}$.

The same catalysts were tested in the cyclopropanation of $\alpha$-methylstyrene (Scheme 1, $\mathrm{R}=\mathrm{CH}_{3}$ ), an alkene with a slightly higher steric hindrance, and the results are summarized in Figure 3. Very similar behaviour was observed.
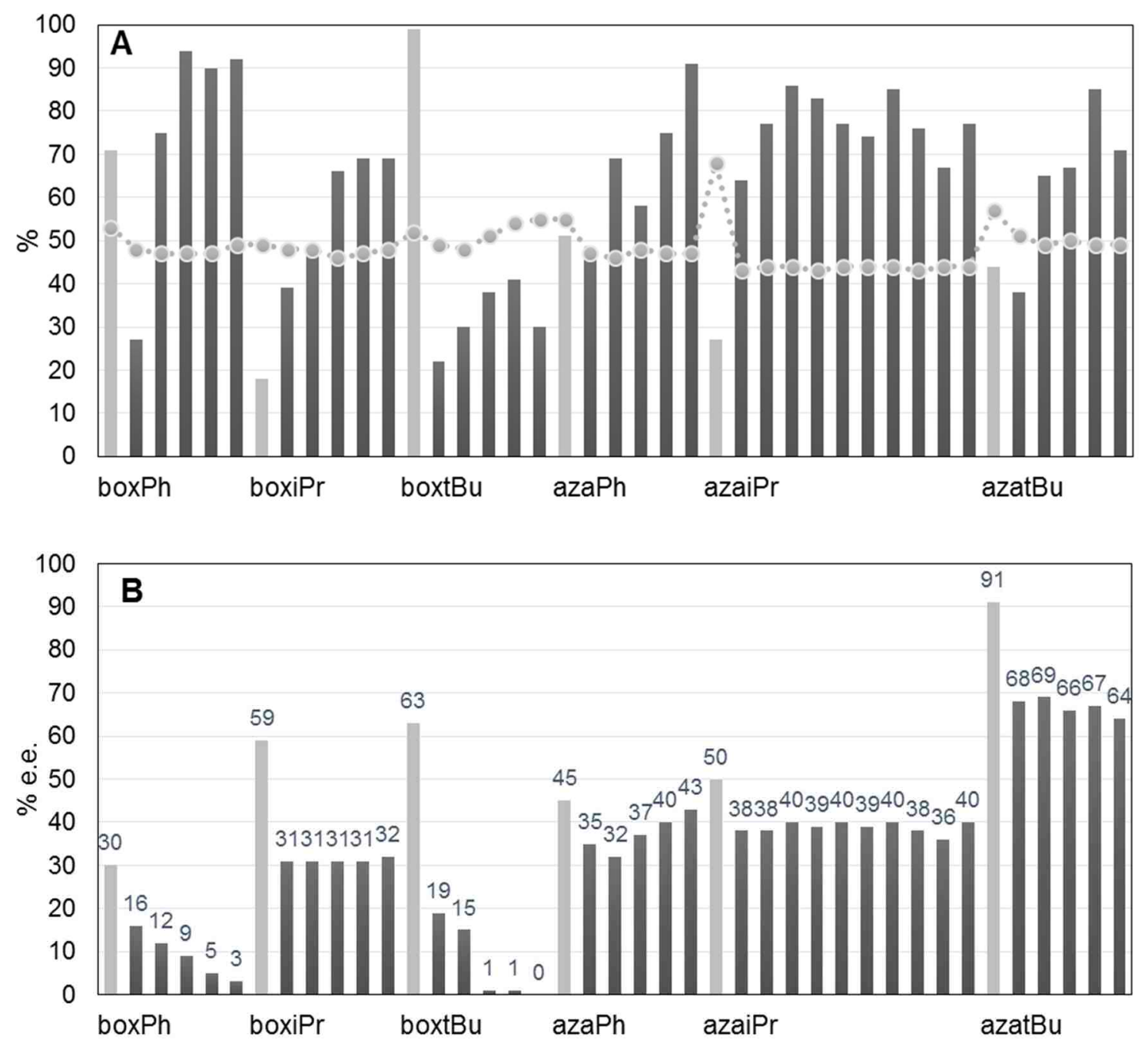

Figure 3. Results of the cyclopropanation of $\alpha$-methylstyrene with ethyl diazoacetate with the six different chiral ligands: in homogeneous phase (grey bars) and in heterogeneous phase (black bars) in succesive runs: A) yield of cyclopropanes in homogeneous phase (grey bars) and in heterogeneous phase (black bars) in succesive runs, and $\%$ of trans cyclopropanes (grey dots), B) enantioselectivity (\% e.e.) of the trans cyclopropanes. 
The yield of cyclopropanes (Figure 3A, bars) was better than in homogeneous phase, and increased from the second re-use, with the only exception being the catalyst modified with box ${ }^{t} \mathrm{Bu}$. The catalyst modified with aza ${ }^{\mathrm{i}} \mathrm{Pr}$ was used for 10 consecutive runs, with yields in the range of $64-86 \%$ (average $76.6 \%$ ). This result represents a productivity of 957 cyclopropane molecules per copper site. In this reaction in the homogeneous phase, the phenyl and methyl groups of $\alpha$-methylstyrene produce a steric hindrance that results in a lower proportion of trans cyclopropane being formed than happens with styrene. Indeed this is mirrored in the results we see with our supported catalysts, and we see that the results are reproduced in the course of the successive recycles with the supported catalysts (Figure 3A, dots).

Regarding enantioselectivity (Figure 3B), the behaviour of the different ligands follows the same trend observed with styrene. The best enantioselectivity is obtained with aza $^{t} \mathrm{Bu}$, with values of $64-69 \%$ e.e. in five consecutive runs. With the other two azabis(oxazolines) the enantioselectivities are lower, but the values are closer to those obtained in solution. For example with aza ${ }^{i} \operatorname{Pr}$ the enantiomeric excess remains stable around $38 \%$ e.e. in the 10 runs. Finally, as in the case of styrene, the box $\mathrm{Ph}$ and boxtBu ligands produce lower enantioselectivities that decrease upon reuse.

\section{Conclusions}

The chiral bis(oxazoline)- and azabis(oxazoline)-copper complexes can be efficiently immobilized on Al-MCM41 by a three-step procedure, consisting of the incipient wetness deposition of a methanol solution of copper(II) triflate on the support, followed by thermal treatment in a flow of air and then modification with the chiral ligand also by incipient wetness impregnation. All of our supported catalysts are active in the cyclopropanation of styrene and $\alpha$-methylstyrene with ethyl diazoacetate, and the behaviour observed is highly dependent on the nature of the ligand. $\mathrm{BoxPh}$ and box ${ }^{\mathrm{t}} \mathrm{Bu}$ ligands lead to very low enantioselectivities that decrease upon recovery. On the contrary, the catalysts prepared with box ${ }^{i} \mathrm{Pr}$, aza ${ }^{i} \mathrm{Pr}$, azaPh and aza ${ }^{t} \mathrm{Bu}$ give results that are stable for at least five consecutive runs. These results show how the stability of the metal complex is a key point that should be taken into account when immobilizing it on a solid support, as it conditions both the efficient immobilization of the complex and the possibility of reuse of the immobilized catalyst. 


\section{Acknowledgement}

This research was made possible by the financial support from the Spanish Ministerio de Economía y Competitividad (Project CTQ2014-52367-R), the European Commission (NANO-HOST program no. PITN-GA-2008-215193) and the Diputación General de Aragón (E11 Group co-financed by the European Regional Development Funds). Dr. Anne Galarneau (Institute Charles Gerhardt, CNRS Montpellier) is gratefully acknowledged for providing the sample of MCM41.

\section{References}

[1] Chiral Catalysts Immobilization and Recycling; D. E. De Vos, I. F. J. Vankelecom, P. A. Jacobs, Eds.; Wiley-VCH: Weinheim, 2000.

[2] H.-U. Blaser, B. Pugin, in Chiral Reactions in Heterogeneous Catalysis, ed. G. Jannes and V. Dubois, Plenum Press, New York, 1995, p. 33.

[3] J. M. Fraile, J. I. García, J. A. Mayoral, Chem. Rev. 109 (2009) 360-417.

[4] P. Kleman, P. Barbaro, A. Pizzano, Green Chem. 17 (2015) 3826-3836.

[5] T. Kawasaki, T. Omine, K. Suzuki, H. Sato, A. Yamagishi, K. Soai, Org. Biomol. Chem. 7 (2009) 1073-1075.

[6] A. Crosman, W. F. Hoelderich, J. Catal. 265 (2009) 229-237.

[7] G. Desimoni, G. Faita, K. A. Jorgensen, Chem. Rev. 111 (2011) PR284-PR437.

[8] J. M. Fraile, M. P. López-Ram-de-Viu, J. A. Mayoral, M. Roldán, J. Santafé-Valero, Org. Biomol. Chem. 9 (2011) 6075-6081.

[9] J. M. Fraile, J. A. Mayoral, A. Muñoz, J. Santafé-Valero, Tetrahedron 69 (2013) $7360-7364$.

[10] J. M. Fraile, N. García, C. I. Herrerías, ACS Catal. 3 (2013) 2710-2718.

[11] J. M. Fraile, J. I. García, C. I. Herrerías, J. A. Mayoral, M. A. Harmer, J. Catal. 221 (2004) 532-540.

[12] Y. Wan, P. McMorn, F. E. Hancock, G. J. Hutchings, Catal. Lett. 91 (2003) 145148.

[13] S. Telalović, U. Hanefeld, Appl. Catal. A 372 (2010) 217-223. 
[14] P. Piaggio, P. McMorn, D. Murphy, D. Bethell, P. C. Bulman Page, F. E. Hancock, C. Sly, O. J. Kerton, G. J. Hutchings, J. Chem. Soc., Perkin Trans. 2 (2000) 2008-2015. [15] S. Taylor, J. Gullick, P. McMorn, D. Bethell, P. C. Bulman Page, F. E. Hancock, F. King, G. J. Hutchings, J. Chem. Soc., Perkin Trans. 2 (2001) 1724-1728.

[16] N. A. Caplan, F. E. Hancock, P. C. Bulman Page, G. J. Hutchings, Angew. Chem., Int. Ed. Engl. 43 (2004) 1685-1688.

[17] R. A. Feldman, J. M. Fraile, Appl. Catal. A 485 (2014) 67-73.

[18] R. A. Feldman, J. M. Fraile, Appl. Catal. A 502 (2015) 166-173.

[19] H. Werner, R. Vicha, A. Gissibl, O. Reiser, J. Org. Chem. 68 (2003) 10166-10168.

[20] A. Galarneau, M. Cangiotti, F. di Renzo, F. Sartori, M. F. Ottaviani, J. Phys. Chem. B 110 (2006) 20202-20210.

[21] J. M. Fraile, J. I. García, C. I. Herrerías, J. A. Mayoral, O. Reiser, A. Socuéllamos, H. Werner, Chem. Eur. J. 10 (2004) 2997-3005.

[22] J. M. Fraile, J. I. García, M. A. Harmer, C. I. Herrerías, J. A. Mayoral, O. Reiser, H. Werner, J. Mater. Chem. 12 (2002) 3290-3295.

[23] J. M. Fraile, J. I. García, C. I. Herrerías, J. A. Mayoral, O. Reiser, M. Vaultier, Tetrahedron Lett. 45 (2004) 6765-6768.

[24] H. Werner, C. I. Herrerías, M. Glos, A. Gissibl, J. M. Fraile, I. Pérez, J. A. Mayoral, O. Reiser, Adv. Synth. Catal. 348 (2006) 125-132.

[25] C. Geiger, P. Kreitmeier, O. Reiser, Adv. Synth. Catal. 347 (2005) 249-254.

[26] L. Aldea, J. M. Fraile, H. García-Marín, J. I. García, C. I. Herrerías, J. A. Mayoral, I. Pérez, Green Chem. 12 (2010) 435-440. 